\title{
Editorial: Smart Objects and Technologies
}

\author{
Marco Furini $^{1} \cdot$ Silvia Mirri $^{2} \cdot$ Kevin Bouchard $^{3} \cdot$ Armir Bujari $^{4}$ \\ Published online: 5 March 2019 \\ (C) Springer Science+Business Media, LLC, part of Springer Nature 2019
}

\section{Editorial:}

Smart objects are entering everyday life and are heavily modifying it. Healthcare, communication, art, entertainment, safety, environment, education, democracy, and human rights, are just a few examples of scenarios that are radically changing thanks to the use of smart objects and technologies.

In this context, the popularity of portable computing devices, such as smartphones, tablets, or smart watches combined with the emergence of many other small smart objects with computational, sensing and communication capabilities coupled with the popularity of social networks and new human-technology interaction paradigms is creating unprecedented opportunities for each of us to do something useful, ranging from a single person to the whole world. Furthermore, Internet of Things, Smart-cities, distributed sensing and Fog computing are representative examples of modern ICT paradigms that aim to describe a dynamic and globally cooperative infrastructure built upon objects intelligence and self-configuring capabilities. These connected objects are finding their way into our pockets, vehicles, urban areas and infrastructure, thus becoming the very texture of our society and providing us the possibility, but also the responsibility, to shape it.

This special issue features six selected high quality papers in the fields of IoT technologies, health communication, community networks, online education, artificial intelligence, and wearable sensor networks.

The first article, titled "Smart Campus: fostering the community awareness through an intelligent environment" presents a case study where IoT technologies have been used to design and develop a system able to bring benefit to communities of students, making them actively involved as central players of such an intelligent environment.

Marco Furini

marco.furini@unimore.it

1 University of Modena and Reggio Emilia, Modena, Italy

2 University of Bologna, Bologna, Italy

3 Université du Québec en Outaouais, Québec, Canada

4 University of Padua, Padova, Italy
The second article, "Towards Using Scientific Publications to Automatically Extract Information on Rare Diseases" focused on rare diseases and proposed a new software tool for automating the extraction of information related to rare diseases from scientific publications.

The third article titled "A low-cost and low-power messaging system based on the LoRa wireless technology" described a low-cost and low-power consumption messaging system based on LoRa technology. The proposed system falls in the category of community networks, where users build their own network where no commercial infrastructure is available.

The fourth article titled "An Online Education System to Produce and Distribute Video Lectures" focused on online learning and the authors shared their experience building ONELab, a system designed to capture, record, edit and stream video lectures. The system supported 1251 students and produced $2064 \mathrm{~h}$ of video lectures, and its evaluation showed that students who used ONELab acquired more credits $(+119 \%)$ and had better grades $(+9.4 \%)$ than those who did not use the system.

The fifth article titled "Intelligent and good machines? The role of domain and context codification" reported reflections and technical aspects of Artificial Intelligence technologies. The authors shows that an inaccurate or incomplete codification of the context where the ANNs will have to operate may result into AI failures.

The last article titled "Safety first? Users' perception of Wearable Sensor Networks for aging", the authors evaluate the acceptability and usability of a system designed to silently monitor the activity and condition of older adults, with a particular focus on the elderly affected by cognitive decline. By using sensors and various wearable objects, the authors' goal is to improve safety and independence of older adults.

Acknowledgements The guest editors are thankful to our reviewers for their effort in reviewing the manuscripts. We also thank the Edit-in-Chief, Dr. Imrich, the Production Coordinators Leonora Mariño Panday and Rolissa Atienza, and the Managing Editor Lucia Zatkova for their supportive guidance during the entire process.

Publisher's Note Springer Nature remains neutral with regard to jurisdictional claims in published maps and institutional affiliations. 


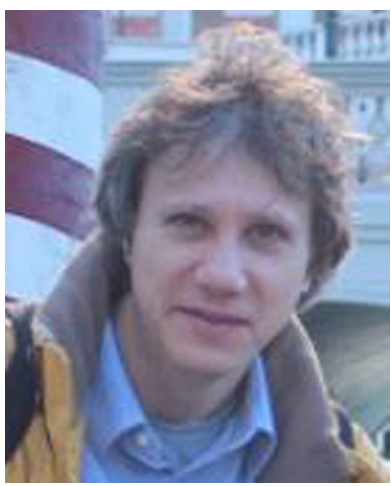

Marco Furini is professor of Computer Science at the University of Modena and Reggio Emilia, Italy. His research interests are multimedia and social computing. In these fields he authored scientific papers published in international journals, conference proceedings, books, and encyclopedia. He holds a US patent related to social media data analysis and he is Associate Editor of the Multimedia Tools and Application journal (Springer) and of the International Journal of Computers and Applications (Taylor and Francis Press). He served as General Chair, TCP Chair and TCP members in different IEEE/ACM international conferences.

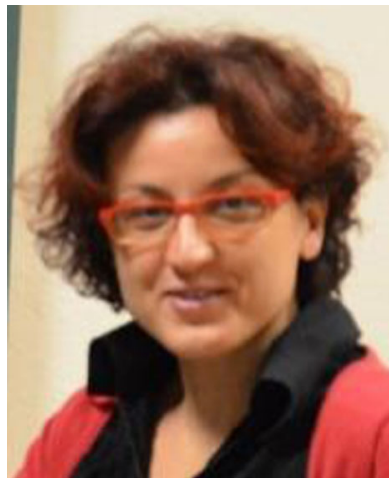

Silvia Mirri received the M.S. and $\mathrm{Ph}$.D. degrees in Computer Science from the University of Bologna, Italy, in 2003 and 2007 respectively. From 2008, she is an assistant professor at the Department of Computer Science and Engineering at the University of Bologna (Italy). In 2008, she was a Visiting Researcher at the University of Toronto (Ontario, Canada). She authored more than 100 publications and she serves as an organization and program committee member for numerous international conferences and workshops. Her research interests focus on Human Computer Interaction, Accessibility, Multimedia and Web applications and technologies.

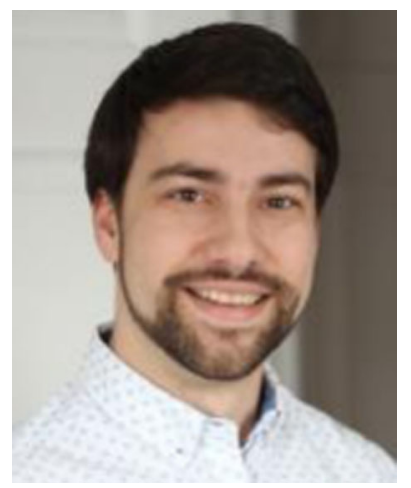

Kevin Bouchard received a $\mathrm{PhD}$ from the Université du Québec en Outaouais (Canada) in 2014, and did a postdoc at University of California Los Angeles (USA) to conceive and implant an ambient system for rehabilitation of patients who suffered from cancer. Since 2016, he is an assistant professor at the Université du Québec à Chicoutimi (Canada) in the computer science department. He authored more than 60 publications and he received funding from several research agencies and companies. His research mostly focus on ambient intelligence, smart homes, assistive technologies, applied AI, and applied machine learning.

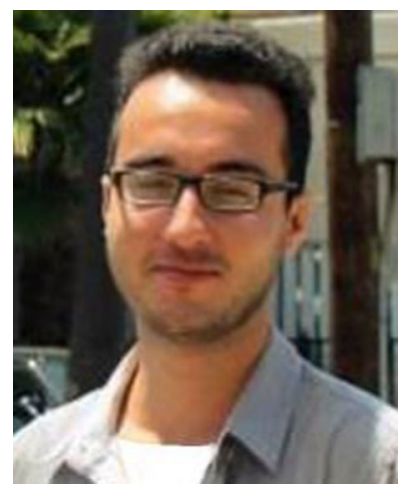

Armir Bujari is an Assistant Professor of Computer Science at the University of Padua. He received his Ph.D. degree in Computer Science at the University of Bologna, Italy, in 2014. His research interests include protocol design and analysis for wireless networks, next generation network architectures and delay-tolerant, mobile communication networks. On these topics, he is active in several technical program committees in international conferences and is coauthor of more than 60 papers, published in international conferences proceedings, books, and journals. 